\title{
Evaluation of mini-FLOTAC method for diagnosing intestinal parasitic infections
}

\section{Original \\ Article}

\author{
Nada A El-Nadi, Amal M Ahmed, Noha S Ahmed, Amany A Abd El-Laah
}

Medical Parasitology Department, Faculty of Medicine, Sohag University, Egypt

\begin{abstract}
Background: Intestinal parasitic infections (IPIs) are widely distributed throughout the world and have been identified as one of the most significant causes of illnesses and diseases. Accurate diagnosis is essential for proper management and to guide the design, implementation and monitoring of programs for community control of infectious diseases.

Objective: To evaluate and compare mini-FLOTAC technique with the widely used formol-ethyl-acetate concentration method (FECM) in IPIs detection.

Material and Methods: One hundred fecal samples were randomly collected from different laboratories. All samples were microscopically examined using mini-FLOTAC and FECM. Iodine and modified Zeil Neelsen stains were used to confirm the presence of protozoa cysts, and oocysts of Cryptosporidium spp. and C. cayetanensis, respectively.

Results: About two thirds of samples (68\%) were positive; 70.6\% (48/68) contained only one parasitic infection by at least one method. Generally, the most frequent was Cryptosporidium spp. 35.3\% (24/68) followed by E. histolytica/dispar $23.5 \%(16 / 68)$ and G. intestinalis $14.7 \%(10 / 68)$. It was observed that mini-FLOTAC detected the highest number of helminthes infections (100\% sensitivity) in contrast to $41.1 \%$ for protozoa whereas FECM was the most sensitive approach for protozoa infections (98.2\% sensitivity) in contrast to $68.7 \%$ for helminthes.

Conclusion: Mini-FLOTAC is a safe rapid device for microscopic examination of stool samples with high sensitivity, affordability, and appropriateness of diagnosis in resource-limited settings where IPIs are widespread.
\end{abstract}

Keywords: Formol-ethyl-acetate concentration, intestinal parasitic infections, mini-FLOTAC.

Received: 29 May, 2019, Accepted: 3 August, 2019.

Corresponding Author: Noha S Ahmed, Tel.: +20 1273126001; E-mail: nohasammer@yahoo.com

Print ISSN: 1687-7942, Online ISSN: 2090-2646, Vol. 12, No. 2, Ausgust, 2019.

\section{INTRODUCTION}

Growing awareness of the clinical importance and public health relevance of IPIs ${ }^{[1,2]}$ induced the need for sensitive inexpensive and simply applied diagnostic tools ${ }^{[3]}$. A wide variety of laboratory methods including direct wet mount, concentration, molecular and culture approaches have been developed for the diagnosis of IPS $^{[4]}$. It was emphasized that an ideal diagnostic test should be of low-cost, and capable of quick and reliable detection of parasites, so that appropriate treatment can be prescribed ${ }^{[5]}$.

The FECM stool concentration technique is the preferred method for qualitative diagnosis of helminthes eggs and protozoa cysts in many clinical parasitology laboratories. This method has the advantage of being rapid and suitable for fresh or preserved stool. However, it requires the availability of centrifugation equipment which may not available in some laboratories ${ }^{[6]}$. Later, fecal egg count (FEC) techniques, such as the FLOTAC were used for qualitative and quantitative diagnosis of helminthes in stools ${ }^{[7]}$. Despite the high sensitivity of FEC and FECM techniques, they are limited by the need for centrifugation of the sample ${ }^{[8]}$. The FLOTAC method, originally developed for diagnosis of veterinary parasites infections ${ }^{[9]}$, was later used for the diagnosis of human intestinal helminthes and protozoa infections $^{[10,11]}$. The mini-FLOTAC technique was then introduced as a reasonable development of FLOTAC for performing multivalent, and qualitative diagnosis of intestinal parasitic infections in human and animal feces. This technique was found to be useful for processing large numbers of samples requiring rapid laboratory diagnosis ${ }^{[12]}$. It has been developed with the aim of combining sensitivity and low cost in order to allow laboratories in resource-limited settings to rely on a good method both for diagnostic and epidemiological purposes $^{[13]}$. The logistic advantages of mini-FLOTAC are that it can be performed on fresh and fixed stool samples, only requires 10-12 min of preparation before microscopic analysis and overcomes the need of a centrifugation step needed in FECM and other concentration techniques, which may pose a problem in laboratories of some developing countries ${ }^{[12]}$. Hence, mini-FLOTAC was indicated as an accurate and precise method for diagnosis ${ }^{[14]}$.

The aim of the study is to evaluate and compare the recently developed mini-FLOTAC technique with the widely used FECM in IPIs detection.

\section{MATERIAL AND METHODS}

This descriptive analytical study was conducted from March 2017 to March 2018. All parasitological 
procedures were conducted in the research laboratory of Medical Parasitology Department, Faculty of Medicine, Sohag University.

Stool samples: One hundred fecal samples were randomly collected from the laboratories of Sohag University Hospital. Each stool sample was preserved in $5 \%$ formalin for mini-FLOTAC and $10 \%$ for FECM, and prepared for applying both FECM according to Garcia $^{[15]}$ and mini-FLOTAC according to Cringoli et $a .^{[16]}$.

Mini-FLOTAC: The mini-FLOTAC apparatus is composed of a base and a reading disc with two $1-\mathrm{ml}$ flotation chambers. It is designed for optimal examination of fecal sample suspensions in each flotation chamber (total volume $=2 \mathrm{ml}$ ). Two miniFLOTAC procedures were performed for each sample as described in FLOTAC protocols ${ }^{[13]}$. Primarily, two grams of stool were placed in the fill-FLOTAC disposable sampling devices composed of a container, a collector and a filter. The sample was diluted with two $\mathrm{ml} 5 \%$ formalin, thoroughly homogenized, and filtered. To the filtrate in two separate tubes, $18 \mathrm{ml}$ of each of two flotation solutions (FS ${ }^{[17]}$, namely FS2 (saturated sodium chloride, specific gravity $(S G)=1.20$; and FS7 (zinc sulphate, $S G=1.35$ ) were added. The FS2 solution was used for the diagnosis of helminthes, the FS7 solution was used for intestinal protozoa ${ }^{[16]}$. The reading disc was examined after $10 \mathrm{~min}$ to allow the eggs to float ${ }^{[13]}$.

Stains: Sample smears were prepared from FECM sediment and stained by iodine and modified Zeil Neelsen stains $^{[15]}$ for the respective identification of E. histolytica/dispar and G. intestinalis cysts, and
Cryptosporidium spp. and C. cayetanensis oocysts suspected in mini-FLOTAC. Slides were examined by 10X, 40X and 100X objectives.

Statistical analysis: Data were analyzed using IBM SPSS statistics for Windows version 25. Chi square test was used to compare the frequency data. Calculation of sensitivity, specificity, and diagnostic accuracy, positive and negative predictive values (PPV and NPV) was done for each method. The results were considered significant when $P$ value $=$ or $<0.05$, and a $95 \%$ CI was calculated. To determine the performance of miniFLOTAC in terms of sensitivity and specificity, FECM was considered the gold standard test.

Ethical considerations: The study was authorized by the Scientific Ethics Committee of the Faculty of Medicine, Sohag University. Consent was obtained individually from all participants included in the study. All infected patients were provided with appropriate treatment.

\section{RESULTS}

Of 100 stool samples examined using both FECM and mini-FLOTAC techniques, $68 \%(68 / 100)$ proved to be positive by at least one method. Among them, $70.6 \%$ $(48 / 68)$ were found to have single infection, $19.1 \%$ $(13 / 68)$ had double infection and 10.3\% (7/68) had three or more infections.

Table (1) shows that in the positive samples, the percentage of detected protozoa was higher than that of helminthes ( $56 \%$ vs $12 \%$ ) with a significant difference $(P=0.03)$. In descending order, frequency of parasitic

Table 1. Results of protozoa and helminthes detection by FECM and mini-FLOTAC methods (No. = 100).

\begin{tabular}{|c|c|c|c|c|c|}
\hline & \multirow{3}{*}{$\begin{array}{l}\text { Total number } \\
\text { detected by a } \\
\text { single method }\end{array}$} & \multirow{2}{*}{ FECM } & \multicolumn{2}{|c|}{ Mini-FLOTAC } & \multirow{5}{*}{$\begin{array}{c}\text { Statistical analysis } \\
\text { Protozoa vs helminthes: } P=0.03^{*} \\
\text { FECM vs mini-FLOTAC: } \\
\text { Protozoa (FEMC): } P=0.01^{*} \\
\text { Helminthes (Mini-FLOTAC): } P=0.01^{*}\end{array}$} \\
\hline & & & $\mathrm{NaCl}(\mathrm{FS} 2)$ & $\mathrm{ZnSO}_{4}$ (FS7) & \\
\hline & & No. $(\%)$ & No. $(\%)$ & No. (\%) & \\
\hline Protozoa & 56 & $55(98.2)$ & $4(7.1)$ & $23(41.1)$ & \\
\hline Helminthes & 12 & $8(75.0)$ & $12(100.0)$ & $7(58.3)$ & \\
\hline \multicolumn{6}{|c|}{ Parasites } \\
\hline Cryptosporidium spp. & 24 & $24(100.0)$ & $1(4.2)$ & $6(25.0)$ & \multirow{6}{*}{$\begin{array}{c}\text { Protozoa } \\
\text { FS7 vs FS2 } \\
P>0.001^{*}\end{array}$} \\
\hline E. histolytica/dispar & 16 & $14(75.0)$ & $1(6.25)$ & $7(43.75)$ & \\
\hline G. intestinalis & 10 & $9(90.0)$ & $1(10.0)$ & $2(20.0)$ & \\
\hline C. cayetanensis & 7 & $7(100.0)$ & $0(0.0)$ & $4(57.1)$ & \\
\hline Blastocystis spp. & 2 & $2(100.0)$ & $0(0.0)$ & $0(0.0)$ & \\
\hline \multirow[t]{2}{*}{ I. belli } & 1 & $1(100.0)$ & $1(100.0)$ & $1(100.0)$ & \\
\hline & otal & & $4(7.1)$ & $23(41.1)^{@}$ & \\
\hline H. nana & 7 & $5(71.4)$ & $7(100.0)$ & $6(85.7)$ & Helminthes \\
\hline A. lumbricoides & 2 & $2(100.0)$ & $2(100.0)$ & $1(50.0)$ & FS2 vs FS7 \\
\hline \multirow[t]{2}{*}{ E. vermicularis } & 3 & $2(66.6)$ & $3(100.0)$ & $1(33.3)$ & $P>0.001^{*}$ \\
\hline & otal & & $12(100.0)$ & $7(58.3)^{@}$ & \\
\hline
\end{tabular}

* Significant, Chi square test was used in statistical analysis.

@ Sum is different from sum of recorded numbers due to presence of mixed infections. 
infections was Cryptosporidium spp. 35.3\% (24/68), followed by $E$. histolytica/dispar 23.5\% (16/68), G. intestinalis $14.7 \%(10 / 68), C$. cayetanensis and $H$. nana both $10.3 \%$ (7/68), A. lumbricoides 4.4\% (3/68), Blastocystis spp. and E. vermicularis both 2.9\% (2/68), I. belli $1.47 \%$ (1/68). Of the non-pathogenic parasites E. coli was detected in $14.7 \%(10 / 68)$ of examined samples and $C$. mesnili in $8.8 \%(6 / 68)$. It was observed that FECM detected more protozoa infections than the mini-FLOTAC (98.2\% vs $41.1 \%$ ); while mini-FLOTAC detected more helminthes infections than FECM $(100 \%$ vs $75 \%)$. The result was statistically significant $(P=$ 0.01). Similarly, both helminthes and protozoa showed contradictory frequencies with each flotation solution used in mini-FLOTAC method. More helminthes infections were detected using $\mathrm{NaCl}$ (FS2) than $\mathrm{ZnSO}_{4}$ (FS7) $(100 \%$ vs $58.3 \%)$. In contrast, FS7 flotation detected more protozoa infections $(41.1 \%)$ than FS2 $(7.1 \%)$. The result was statistically significant $(P>$ 0.001). Regarding Cryptosporidium spp. FECM detected
$100 \%$ infections, while mini-FLOTAC FS7 detected only $25 \%$. Similarly, detection of $E$. histolytica/dispar was $87.5 \%$ vs $43.75 \%$; $G$. intestinalis was $90 \%$ vs $20 \%$; and $C$. cayetanensis was $100 \%$ vs $57.1 \%$. In contrast for helminthes, FECM and mini-FLOTAC FS2 and FS7 showed more or less similar results for detection of $H$. nana $(71.4 \%, 100 \%$, and $85.7 \%$, respectively), but was insignificantly higher with mini-FLOTAC than FECM.

Calculation of sensitivity, specificity, PPV, NPV and diagnostic accuracy revealed that mini-FLOTAC FS2 is more sensitive than FECM (100\% vs $66.7 \%)$, with better diagnostic accuracy (100\% vs 96\%) in diagnosis of helminthes infections (Table 2). In protozoa infections, FECM proved more sensitive than mini-FLOTAC $(98.2 \%$ vs $41.1 \%$ ), but mini-FLOTAC FS2 was more specific than FECM (100\% vs 57\%), with better diagnostic accuracy of FECM (99\%), followed by mini-FLOTAC FS7 (67\%) then mini-FLOTAC FS2 (48\%).

Table 2. Calculated parameters for evaluation of mini FLOTAC and FECM in diagnosis of helminthes and protozoa.

\begin{tabular}{|c|c|c|c|c|c|}
\hline & Sensitivity\% & Specificity $\%$ & PPV\% & NPV\% & Diagnostic accuracy $\%$ \\
\hline \multicolumn{6}{|l|}{ Helminthes } \\
\hline FECM & 66.7 & 100.0 & 85.7 & 95.7 & 96.0 \\
\hline Mini-FLOTAC & 100.0 & 95.6 & 66.7 & 100.0 & 100.0 \\
\hline $\mathrm{NaCl}$ (FS2) & 100.0 & 95.6 & 66.7 & 100.0 & 100.0 \\
\hline $\mathrm{ZnSO}_{4}$ (FS7) & 58.3 & 98.9 & 85.7 & 94.6 & 95.0 \\
\hline \multicolumn{6}{|l|}{ Helminthes } \\
\hline FECM & 98.2 & 57.7 & 40.0 & 97.8 & 99.0 \\
\hline Mini-FLOTAC & 41.1 & 97.7 & 95.6 & 57.1 & 67.0 \\
\hline $\mathrm{NaCl}$ (FS2) & 7.1 & 100.0 & 100.0 & 45.8 & 48.0 \\
\hline $\mathrm{ZnSO}_{4}(\mathrm{FS} 7)$ & 41.1 & 97.7 & 95.6 & 57.1 & 67.0 \\
\hline
\end{tabular}

PPV: Positive predictive value, NPV: Negative predictive value,

\section{DISCUSSION}

IPIs signify health problems, particularly among children in developing countries, causing significant morbidity and mortality, and have been termed as "the cancers of developing nations ${ }^{[18]}$. Children being the best indicators of prevalence of IPIs, a research study from Egypt reported that $27 \%$ of examined children were infected with intestinal parasites ${ }^{[19]}$. The researchers suggested that development of effective preventive and control measures relies on epidemiological studies to produce baseline data on the prevalence of parasitic infections. Such data invariably depend on the diagnostic techniques employed where the field of diagnostic medical parasitology is undergoing dramatic change. In recent years, tremendous efforts concentrated on research for the development of newer diagnostic methods focusing on serological, molecular, and proteomic approaches $^{[20]}$. Direct methods for the diagnosis of parasitic infections that include microscopy continue to be the routine in most diagnostic laboratories. Hence, the call for development of more rapid tests that do not affect sensitivity and that can be employed in clinical locations as well as in field settings with poor resources. Needless to say, accurate diagnosis is essential for implementation of cost-effective control measures and for mapping, impact evaluation, and surveillance of IPIs ${ }^{[21,22]}$. Hence, the crucial need for reliable, sensitive and practical diagnostic methods. Mini-FLOTAC was created with the concept of matching good sensitivity with a low-cost technique. This prompted us to evaluate it in comparison with FECM for qualitative diagnosis of IPIs and as a tool for surveillance and epidemiological studies.

On the whole, examination of 100 stool samples collected randomly from different laboratories in Sohag governorate showed that $68 \%$ were positive for protozoa and helminthes by at least one method. Protozoa were apparently more frequently detected than helminthes parasitizing $56 \%$ vs $12 \%$ of the positive samples respectively, with a significant difference $(P=0.03)$. It was observed that FECM detected more protozoa infections than mini-FLOTAC $(98.2 \%$ vs $41.1 \%)$, while mini-FLOTAC detected more helminthes infections than FECM (100\% vs $75 \%)$. 
This was significant $(P=0.01)$, and agreed with other studies, in which mini-FLOTAC was the most sensitive method for detecting helminthes infections compared with the FECM for diagnosis in humans ( $90 \%$ vs $60 \%)$, whereas FECM was the most sensitive for intestinal protozoa infections ( $88 \%$ vs $68 \%)^{[12]}$. In another report, mini-FLOTAC technique displayed $83.3 \%$ and $100 \%$ detection of some eggs of trematodes and nematodes infections, respectively ${ }^{[23]}$. In our study FECM recorded a higher rate of infection by Cryptosporidium spp. $(100 \%)$ as compared to that detected by mini-FLOTAC $(25 \%)$. The second most prevalent intestinal parasite identified in this study also by FECM was E. histolytica/ dispar (87.5\%) as compared to mini-FLOTAC (43.75\%).

Microscopic diagnosis of intestinal protozoa in fresh stool samples is more difficult, requiring special staining and experienced highly skilled technicians for correct identification and differentiation of protozoa stages. It is more time consuming than the microscopic detection of helminthes eggs in human stool samples ${ }^{[17]}$. The sensitivity of microscopy can be increased by reducing the debris, which is achieved by concentration from feces. Common methods used for the concentration are centrifugation, and FECM is more widely used as it is more sensitive ${ }^{[24]}$. Diagnostic visualization of Cryptosporidium and Cyclospora oocysts can be achieved by a number of techniques including microscopic examination by staining the smears with modified acid-fast stain ${ }^{[25,26,24]}$. D'Antoni's iodine has been found to be better for detection of cysts of Entamoeba and Giardia in fresh stool smears ${ }^{[27]}$.

Concentration of fecal specimens is principally based on the differences in specific gravity of the solution and the parasites and removal of fecal debris in the background, enabling the detection of scanty organisms ${ }^{[28]}$. It was noted that the flotation procedure yields a clear preparation due to separation of protozoan cysts, coccidian oocysts and certain helminthes eggs and larvae from excess debris through the use of solutions with a high specific gravity ${ }^{[29]}$. Cryptosporidium spp. was detected in $100 \%$ by FECM vs $25 \%$ by mini-FLOTAC; and E. histolytica/dispar recorded $87.5 \%$ vs $43.75 \%$, respectively. G. intestinalis and $C$. cayetanensis rates were $90 \%$ and $100 \%$ by FECM and with lower percentages by mini-FLOTAC $(20 \%$ and $57.1 \%$, respectively). Results recorded by Becker et al. $^{[17]}$ presented higher prevalence of protozoa by FLOTAC-400 dual technique in contrast to FECM. Their study proved that the prevalence of $E$. coli was respectively $77.8 \%$ vs $71.3 \%, P<0.001 ; B$. hominis was $20.4 \%$ vs $13.0 \%, P=0.458$; and $G$. intestinalis was $8.3 \%$ vs $6.5 \%, P<0.001$ ); the FECM detected higher prevalence of $E$. histolytica/dispar $(27.8 \%$ vs $20.4 \%, P=$ $0.019)$. The researchers concluded that changes in the specific gravity of the solutions may play an important role in the visualization of the parasites ${ }^{[17]}$. However, the poor performance for protozoan detection by miniFLOTAC in our current study may be that the use of
400X at maximum did not allow perfect visibility of internal structures. This observation was noticed also by Barda et al. ${ }^{[12]}$.

Concerning the use of flotation solutions of different specific gravities, we observed that FS7 $\left(\mathrm{ZnSO}_{4}\right)$ apparently gave a more significant detection of protozoa stages than $\mathrm{FS} 2(\mathrm{NaCl})$ ( $41.1 \%$ vs $7.1 \%$, respectively) $(P>0.001)$; and was more sensitive in detection of $C$. cayetanensis, E. histolytica/dispar, Cryptosporidium spp., and $G$. intestinalis $\quad(57.1 \%, 43.7 \%, 25 \%, 20 \%$ respectively). Except for $I$. belli, FS2 detection ratio of these protozoa species was insignificant and can be neglected, considering it unsuitable for protozoa diagnosis. It may be attributed to the crucial role of specific gravity of the solution in flotation of the parasites. From our results it is apparent that the miniFLOTAC FS2 is the most sensitive approach (100\%) for helminthes diagnosis, while FECM is more sensitive for protozoa diagnosis (98.2\%). In agreement the study done by Barda et al. ${ }^{[7]}$ confirmed that the miniFLOTAC sensitivity for helminthes was $95 \%$ and FECM sensitivity for protozoa was $88 \%$. Also, our results are close to the study done by Hussein et al. $^{[3]}$ in which mini-FLOTAC sensitivity for helminthes was $100 \%$ and FECM sensitivity for protozoa was $100 \%$. An important observation is that the diagnostic accuracy of the miniFLOTAC changes according to the FS used. In our study helminthes were more accurately diagnosed by FS2 $(100 \%)$, while protozoa stages were diagnosed more by FS7 (67\%). Result regarding effect of flotation solutions on helminthes infections was reversed showing apparent better detection by FS2 than FS7 (100\% vs $58.3 \%$ ). H. nana recorded $100 \%$ and $85.7 \%$ by FS2 and FS7, respectively. This agrees with several studies $^{[20,22,30]}$ which detected higher sensitivity of FS2 in helminthes detection. Hussein et al. ${ }^{[3]}$ revealed that FS7 was generally more sensitive (100\%) for helminthes diagnosis but with no significant difference between FS2 and FS7. This disagreed completely with the findings of our study in which FS7 was less sensitive $(58.3 \%)$ in helminthes diagnosis with a significant difference between FS2 and FS7.

Therefore, when considering results of intestinal protozoan infections, recorded by our present study and other studies mentioned here, there is a relatively poor performance of mini-FLOTAC. However, the comparison of mini-FLOTAC versus FECM could support the use of mini-FLOTAC in protozoa diagnosis in certain situations, e.g. in areas with heavy parasite load and limited resources, taking in consideration their feasibility and cost-benefits. An advantage of the mini-FLOTAC technique is that it permits work with fixed fecal samples. Formalin or any preservative can be added and the samples stored for further testing ${ }^{[31]}$. This allows the possibility of examining the samples on different days and improves the quality control process. An additional advantage of the fill-FLOTAC-miniFLOTAC system is that it is a "closed" method and the 
system is thoroughly safe with no risk of contamination for the operator. As for the cost of equipment, the only cost for the mini-FLOTAC is the purchase of $\mathrm{NaCl}$ and $\mathrm{ZnSO}_{4}$ for the flotation solutions, and the cost of the kit which is cheap; whereas FECM requires a centrifuge, formol and ethyl-acetate that are not always easy to purchase, especially in laboratories with limited resources. All mini-FLOTAC reading disks and the fillFLOTAC containers are reusable after careful washing.

From the current study, it is concluded that miniFLOTAC can be used to replace FECM for qualitative diagnosis and surveillance of helminthes infections especially in developing countries (including Egypt), whereeconomical facilities maybe a problem,inaddition to the advantage of being highly sensitive, simple, safe, cheap technique and not requiring centrifugation step. While, for diagnosis of intestinal protozoa we suggest FECM as the most sensitive method, considering the low sensitivity of mini-FLOTAC in diagnosis.

Author contribution: AA Abd El-Laah designed the study, shared NS Ahmed in performing the experiments and wrote the manuscript; AM Ahmed and NA El-Nadi shared in research planning and writing the manuscript. All authors critically revised the manuscript.

Conflict of interest: Authors confirm that there are no known conflicts of interest associated with this study.

\section{REFERENCES}

1. Hotez PJ, Brindley PJ, Bethony JM, King CH, Pearce EJ, Jacobson J. Helminth infections: the great neglected tropical diseases. J Clin Invest 2008; 118:1311-1321.

2. Ouattara M, Silué KD, N' Guéssan AN, Yapi A, Barbara $\mathrm{M}$, Raso G, et al. Prevalence and polyparasitism of intestinal protozoa and spatial distribution of Entamoeba histolytica, E. dispar and Giardia intestinalis from pupils in the rural zone of Man in Côte d'Ivoire. Santé 2008; 18:215-222. (In French)

3. Hussein AH, Rashed SM, El-Hayawan IA, Aly NSM, Abou Ouf EA, Ali AT. Intestinal parasitic infections and accuracy of direct thin and thick smear, formolether sedimentation, centrifugal flotation, and mini-FLOTAC techniques among patients with gastrointestinal tract disorders from the greater Cairo region, Egypt. Am J Trop Med Hyg 2017; 96(3):589-594.

4. Garcia LS. Diagnostic Medical Parasitology. 4th edition. Washington, DC: ASM Press; 2001: 1069.

5. Mwape KE, Gabriël S. The parasitological, immunological, and molecular diagnosis of human taeniasis with special emphasis on Taenia solium taeniasis. Curr Trop Med Rep 2014; 1(4):173-180.

6. Lier T, Simonsen GS, Wang T, Lu D, Haukland HH, Vennervald BJ, et al. Real-time polymerase chain reaction for detection of low-intensity Schistosoma japonicum infections in China. Am J Trop Med Hyg 2009; 81:428-432.

7. Cringoli G, Rinaldi L, Maurelli MP, Utzinger J. FLOTAC: new multivalent techniques for qualitative and quantitative copro-microscopic diagnosis of parasites in animals and humans. Nat Protoc 2010; 5(3):503-515.

8. Knopp S, Glinz D, Rinaldi L, Mohammed KA, N'Goran EK, Stothard JR, et al. FLOTAC: A promising technique for detecting helminthes eggs in human feces. Royal Soc Trop Med Hyg 2009; 103(12):11901194.

9. Cringoli, GL, Rinaldi M, Albonico R, Bergguist R, Utzinger J. Geospatial (s) tools: integration of advanced epidemiological sampling and novel diagnostics. Geospatial Health 2013;7:399-404.

10. Rinaldi l, Amadessi A, Dufourd E, Chartier C, Bosco A, Gadanho $\mathrm{M}$ et al. Rapid assessment of faecal egg count and faecal egg count reduction through composite sampling in cattle. Parasite Vectors 2019; 12(1): 353.

11. Cringoli GL, Rinaldi MP, Maurelli, MP, Morgoglione ME, Musella V, Utzinger J. Ancylostoma caninum: Calibration and comparison of diagnostic accuracy of flotation in tube, McMaster and FLOTAC in fecal samples of dogs. Exp Parasitol 2011; 128: 32-37.

12. Barda BD, Rinaldi L, Ianniello D, Zepherine $H$, Salvo F, Sadutshang T. Mini-FLOTAC, an innovative direct diagnostic technique for intestinal parasitic infections: Experience from the Field. PLoSNegl Trop Dis 2013; 7(8): 2344.

13. Barda B, Cajal P, Villagran E, Cimino R, Juarez M, Krolewiecki A et al. Mini-FLOTAC, Kato-Katz and McMaster: Three methods, one goal; highlights from north Argentina. Parasit Vectors. 2014; 7(1): 271.

14. Paras KL, George MM, Vidyashankar AN, Kaplan RM. Comparison of fecal egg counting methods in four livestock species. Vet Parasitol 2018;257:2127.

15. Garcia LS. Diagnostic Medical Parasitology, $6^{\text {th }}$ Edition 2016.

16. Cringoli G, Maurelli MP, Levecke B, Bosco A, Vercruysse J, Utzinger J, et al. The Mini-FLOTAC technique for the diagnosis of helminth and protozoan infections in humans and animals. Nat Protoc 2017; 12(9):1723-1732.

17. Becker SL, Lohourignon LK, Speich B, Rinaldi L, Knopp S, Ngoran EK et al. Comparison of the Flotac-400 dual technique and the FECT for diagnosis 51 of human intestinal protozoon infection. J Clinic Microbiol 2011; 49(6):21832190.

18. Al-Delaimy AK, Al-Mekhlafi HM, Nasr NA, Sady H, Atroosh WM, Nashiry M, et al. Epidemiology of intestinal polyparasitism among Orang Asli school children in rural Malaysia. PLoS Negl Trop Dis 2014; 8(8): e3074.

19. Monib MEM, Hassan AAAE, Attia RAEH, Khalifa MM. Prevalence of intestinal parasites among children 
attending Assiut university children's hospital, Assiut, Egypt. J Adv Parasitol 2016; 3(4):125-131.

20. Ricciardi A, NdaoM. Diagnosis of parasitic infections: What's Going On? Sage journals 2015; 20(1); 6-21.

21. Bundy DAP, Hall A, Medley GF, Savioli L. Evaluating measures to control intestinal parasitic infections. World Health Stat 1992; 45:168-179.

22. Nikolay B, Brooker SJ, Pullan RL. Sensitivity of diagnostic tests for human soil-transmitted helminth infections: A meta-analysis in the absence of a true gold standard. Int J Parasitol 2014; 44(11):765-774.

23. Alvarado-Villalobos MA, Cringoli G, Maurelli MP, Cambou A, Rinaldi L, Barbachano-Guerrero A, et al. Flotation techniques (FLOTAC and mini-FLOTAC) for detecting gastrointestinal parasites in howler monkeys. Parasit Vectors 2017; 10(1):586.

24. Khurana S and Chaudhary P. Laboratory diagnosis of cryptosporidiosis. Trop Parasitol. 2018; 8:2-7.

25. Visvesvara GS, Moura H, Kovacs-Nace E, Wallace $\mathrm{S}$, Eberhard ML. Uniform staining of Cyclospora oocysts in fecal smears by a modified safranin technique with microwave heating. J Clin Microbiol 1997; 35(3):730-733.
26. Yazar S, Yalcin S,Sahin I. Human cyclosporiosis in Turkey. World J Gastroenterol 2004;10:1844-1847.

27. Shetty N, Prabhu T. Evaluation of fecal preservation and staining methods in the diagnosis of acute amoebiasis and giardiasis. J Clin Pathol 1988; 41:694-699.

28. McHardy IH, Wu M, Shimizu-Cohen R, Couturier MR, Humphries RM. Detection of intestinal protozoa in the clinical laboratory. J Clin Microbiol 2014; 52:712-720.

29. O'Horo M, Comstock A, Hoffmaster L, Hunter A, Jones R, Lloyd $\mathrm{R}$ et al. A comparison of fecal examination techniques for the recovery of parasite ova in large animal. Vet Tech 2007; 28: 442-443.

30. Assefa LM, Crellen T, Kepha S, Kihara JH, Njenga SM, Pullan RL, Brooker SJ. Diagnostic accuracy and cost-effectiveness of alternative methods for detection of soil-transmitted helminths in a posttreatment setting in western kenya. PLoS Neg Trop Dis 2014; 8(5):2843.

31. Capasso M, Maurelli MP, Ianniello D, Alves LC, Amadesi A, Laricchiuta $\mathrm{P}$, et al. Use of mini-FLOTAC and Fill-FLOTAC for rapidly diagnosing parasitic infections in zoo mammals. Rev Bras Parasitol Vet 2019;28(1):168-171. 